\title{
Drop size distribution comparisons between Parsivel and 2-D video disdrometers
}

\author{
M. Thurai ${ }^{1}$, W. A. Petersen ${ }^{2}$, A. Tokay ${ }^{3}$, C. Schultz ${ }^{4}$, and P. Gatlin ${ }^{4}$ \\ ${ }^{1}$ Colorado State University, Fort Collins, Colorado, USA \\ ${ }^{2}$ NASA/MSFC, Huntsville, Alabama, USA \\ ${ }^{3}$ JCET - University of Maryland Baltimore County, Baltimore, USA \\ ${ }^{4}$ University of Alabama, Huntsville, USA
}

Received: 8 November 2010 - Revised: 11 February 2011 - Accepted: 10 March 2011 - Published: 9 May 2011

\begin{abstract}
Measurements from a 2-D video disdrometer (2DVD) have been used for drop size distribution (DSD) comparisons with co-located Parsivel measurements in Huntsville, Alabama. The comparisons were made in terms of the mass-weighted mean diameter, $D_{\mathrm{m}}$, the standard deviation of the mass-spectrum, $\sigma_{\mathrm{m}}$, and the rainfall rate, $R$, all based on 1-min DSD from the two instruments. Time series comparisons show close agreement in all three parameters for cases where $R$ was less than $20 \mathrm{~mm} \mathrm{~h}^{-1}$. In four cases, discrepancies in all three parameters were seen for "heavy" events, with the Parsivel showing higher $D_{\mathrm{m}}$, $\sigma_{\mathrm{m}}$ and $R$, when $R$ reached high values (particularly above $30 \mathrm{~mm} \mathrm{~h}^{-1}$ ). Possible causes for the discrepancies include the presence of a small percentage of non-fully melted hydrometers, with higher than expected fall velocity and with very different axis ratios as compared with rain, indicating small hail or ice pellets or graupel. We also present here Parsivel-to-Parsivel comparisons as well as comparisons between two 2DVD instruments, namely a low-profile unit and the latest generation, "compact unit" which was installed at the same site in November 2009. The comparisons are included to assess the variability between the same types of instrument. Correlation coefficients and the fractional standard errors are compared.
\end{abstract}

\section{Introduction}

Satellite-based precipitation retrieval algorithms such as those used for the NASA Tropical Rainfall Measurement Mission (TRMM) or those currently under development for

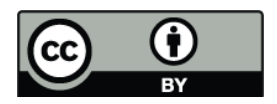

Correspondence to: $\mathrm{M}$. Thurai (merhala@engr.colostate.edu) the Global Precipitation Mission (GPM) require robust 3-D descriptions of rainfall characteristics such as the drop size distribution (DSD) and it's variability over satellite instrument footprints ranging from approximately $5-15 \mathrm{~km}$ (e.g., Hou et al., 2008; Iguchi et al., 2009). The rather instantaneous, bulk nature of a low-earth orbiting satellite measurement (e.g., consider a single TRMM Precipitation Radar swath) integrates DSD spatial variability that occurs at subpixel scales. Moreover, a given orbit will not sample the temporal variability, and, furthermore, errors are introduced due to beam filling effects and the relatively large pixel sizes. To assist in providing the most accurate representation of DSDs and associated error for development of physically consistent retrieval algorithms and the associated higher level moment estimates (i.e., e.g., rainfall rate, radar reflectivity), it is important to quantify pixel to sub-pixel scale variability of the DSD.

Surface based measurements provided by disdrometers provide a means to address the question of DSD and associated moments of the DSD variability. However, these instruments provide only point measurements (if used in typical single-instrument deployments, are expensive and, depending on instrument type, can be difficult to maintain in networks of sufficient instrument density over the footprint scale of interest (i.e., order of 20 instruments at $1-2 \mathrm{~km}$ spacing).

One solution we are proposing to implement for validation activities related to GPM involves a joint deployment of a large number (16-20) of Parsivel disdrometers (LofflerMang and Joss, 2000; Tapiador et al., 2010) anchored by approximately five 2-D Video Disdrometers (Schoenhuber et al., 2008) to sample the spatial and temporal decorrelation properties of the DSD at scales of $1 \mathrm{~km}$ over a satellite footprint scale of $\sim 10 \mathrm{~km}$. These measurements will also be used

Published by Copernicus Publications on behalf of the European Geosciences Union. 
to "bootstrap" the coverage domain of the DSD estimates using dual-polarimetric radar (Bringi et al., 2009). Fundamental to assessing instrument error in any resulting conclusion pertaining to the "true" DSD variability, is the interinstrument disdrometer measurement errors, which should be quantified. As a first step to accomplishing objectives related to the DSD correlation characteristics, herein we address the inter-instrument error measurement characteristics as observed in a range of cold and warm season rainfall systems composed of a wide range of rainfall. Time series comparisons of two DSD parameters and rainfall rate $R$ from two Parsivel and two 2DVD measurements are made and their statistical parameters such as Pearson correlation coefficient and fractional standard errors are evaluated. We also address the issue of variability between the same type of instrument versus the inter-instrumental variability.

\section{Experimental set-up and data}

The two Parsivel disdrometers (OTT) and the two 2-D video disdrometers used in this study are all collocated at a site in Huntsville, Alabama, the site being a heavily instrumented, bermed research area, designed to measure precipitation properties in various rain regimes. Other instruments include a UHF profiler, several rain gauges, including a Geonor weighing gauge, and a C-band dual-polarization radar situated $15 \mathrm{~km}$ away (cf., Petersen et al., 2007). Both the C-band radar and the profiler provide useful information regarding the rain regime and the vertical structure respectively while the Geonor rain gauge provides useful validation for daily rainfall accumulation.

The Parsivel disdrometer is a laser optical device which in theory - can measure the size and fall speed of hydrometeors. The size category goes up to $25 \mathrm{~mm}$, with 32 size classes of varying diameter intervals, and the velocity category goes up to $20 \mathrm{~m} \mathrm{~s}^{-1}$, again with 32 classes, and again with varying velocity intervals. Details of the instrument and the measurement technique, together with the assumptions used to determine the size and velocity of hydrometeors can be found in Loffler-Mang and Joss (2000), Battaglia et al. (2010) and Tapiador et al. (2010). However, there are two main points worth noting for the Parsivel, which are relevant to the present study:

(i) The hydrometeors are assumed to be oblate spheroids, with a "pre-assumed" relationship between drop axis ratio and drop diameter;

(ii) The output of the instrument is contained in a 32 by 32 matrix, with size versus velocity elements; in other words, the data are 'binned' under size and velocity categories (Parsivel is also a real-time weather sensor which provides the precipitation type such as "GR" for graupel).
On the other hand, the 2-D video disdrometer provides information on individual hydrometeors, determined from two camera images taken at orthogonal angles. The two optical planes are offset by a precisely-known distance, and by matching each hydrometeor from the first optical plane to the second, it's fall speed is determined. In the case of rain, such matching procedure is relatively simple because of the rotational symmetry of drop shapes, and, furthermore, for larger drops ( $>2 \mathrm{~mm}$ drop diameter), the procedure becomes even simpler and results in much less errors due to mismatches. Further information can be found in Schoenhuber et al. (2008); Kruger and Krajewski (2002), and Huang et al. (2008).

For DSD comparisons between Parsivel and 2DVD, events with widely varying rain regimes were chosen, ranging from one outer hurricane rain band, to multi-season stratiform rain events, to localized convective cells, and to organized convective squall-lines. For all events, DSD comparisons were performed in terms of the mass-weighted mean diameter $\left(D_{\mathrm{m}}\right)$ and the width of the mass spectrum $\left(\sigma_{\mathrm{m}}\right)$, both calculated numerically from the DSD measured over $1 \mathrm{~min}$. The definitions of the two parameters are given in Ulbrich and Atlas (1998). The rainfall rate, $R$, was also chosen for statistical comparisons, again based on the 1-min DSD. In the case of 2DVD, the three quantities were evaluated on a dropby-drop basis (after ensuring accurate calibration) whereas for the Parsivel disdrometer the "binned" output of the 1-min DSD data were used.

\section{Parsivel versus 2DVD Comparisons}

\subsection{Time series data}

Most of the events considered here were of long duration (at least over $90 \mathrm{~min}$ ). Figure 1a shows the time series of the measured 1-min DSD (notated as N(D) hereafter) for one of the longest events sampled on 6 January 2009; the UTC is from 00:00 UTC to 24:00 UTC, i.e. the entire day. The computed rain rate did not exceed $30 \mathrm{~mm} \mathrm{~h}^{-1}$, except during 2 very short periods. The colour scale in Fig. 1a represents $\mathrm{N}(\mathrm{D})$ in units of $\mathrm{mm}^{-1} \mathrm{~m}^{-3}$ determined from the 2DVD measurements. Despite the long duration, the event is seen to be composed of mostly small and medium sized drops, i.e. $D$ did not exceed $4 \mathrm{~mm}$ for the entire event. The black marks superimposed on the colour plot represent $D_{\mathrm{m}}$ and the grey points represent $\sigma_{\mathrm{m}}$ (evaluated using, for example, Eqs. (3) and (18) in Thurai and Bringi, 2008). The former does not exceed $2 \mathrm{~mm}$ for much of the time, whilst the latter is mostly below $1 \mathrm{~mm}$. The two are compared with the corresponding estimates from one of the Parsivels in Fig. 1b, c respectively, and their conditioned histograms (in terms of percentage probability, conditioned to $D_{\mathrm{m}}>0.1 \mathrm{~mm}$ ) are compared in Fig. 1d, e respectively. In both cases, i.e. time series comparisons and the histogram comparisons, excellent 

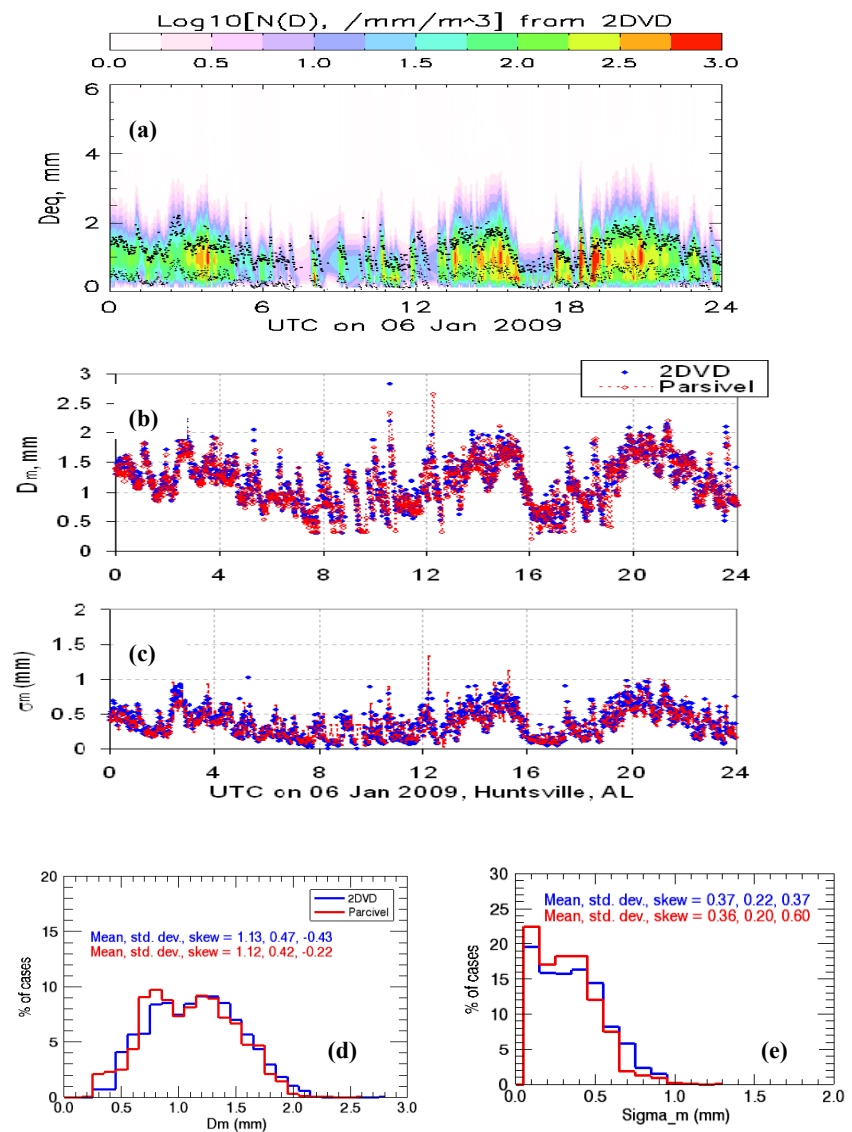

Fig. 1. (a): 1-min DSD from the 2DVD measurements for a $24 \mathrm{~h}$ event; the color scale represents $\log _{10}[\mathrm{~N}(\mathrm{D})]$ where $\mathrm{N}(\mathrm{D})$ is in units of $/ \mathrm{mm} / \mathrm{m}^{3}$; overplotted are $D_{\mathrm{m}}$ (black points) and $\sigma_{\mathrm{m}}$ (grey points) determined from N(D). (b): $D_{\mathrm{m}}$ time series comparisons between 2DVD and Parsivel measurements. (c): the corresponding $\sigma_{\mathrm{m}}$ comparisons. (d and e): comparisons of the conditioned histograms of $D_{\mathrm{m}}$ and $\sigma_{\mathrm{m}}$.

agreement can be seen. To quantify the comparisons, the mean, standard deviation and the skewness of the histograms in Fig. 1d, e are $1.13 \mathrm{~mm}, 0.47 \mathrm{~mm}$ and $0.43 \mathrm{~mm}$ from the 2DVD data, respectively, compared with $1.12 \mathrm{~mm} .0 .42 \mathrm{~mm}$ and $0.22 \mathrm{~mm}$ from the Parsivel data which are very close.

Several other events considered (not shown here) also showed equally good agreement between Parsivel and 2DVD in terms of $D_{\mathrm{m}}$ and $\sigma_{\mathrm{m}}$ time series. The events included the remnants of a hurricane (Faye in 2009), with high wind speeds accompanied by significant gusts. However, there were four events (out of all the events considered) which showed noticeable discrepancies within the event durations. One example is shown in Fig. 2a, b. For about $30 \mathrm{~min}$ at the beginning of the event, the agreement between Parsivel and the 2DVD is very good (both in $D_{\mathrm{m}}$ and $\sigma_{\mathrm{m}}$ ), but in the following hour, discrepancies are seen in both variables, with Parsivel showing higher $D_{\mathrm{m}}$ and higher $\sigma_{\mathrm{m}}$ during this period. Thereafter, the time series reverts to being in close agreement between the two instruments.
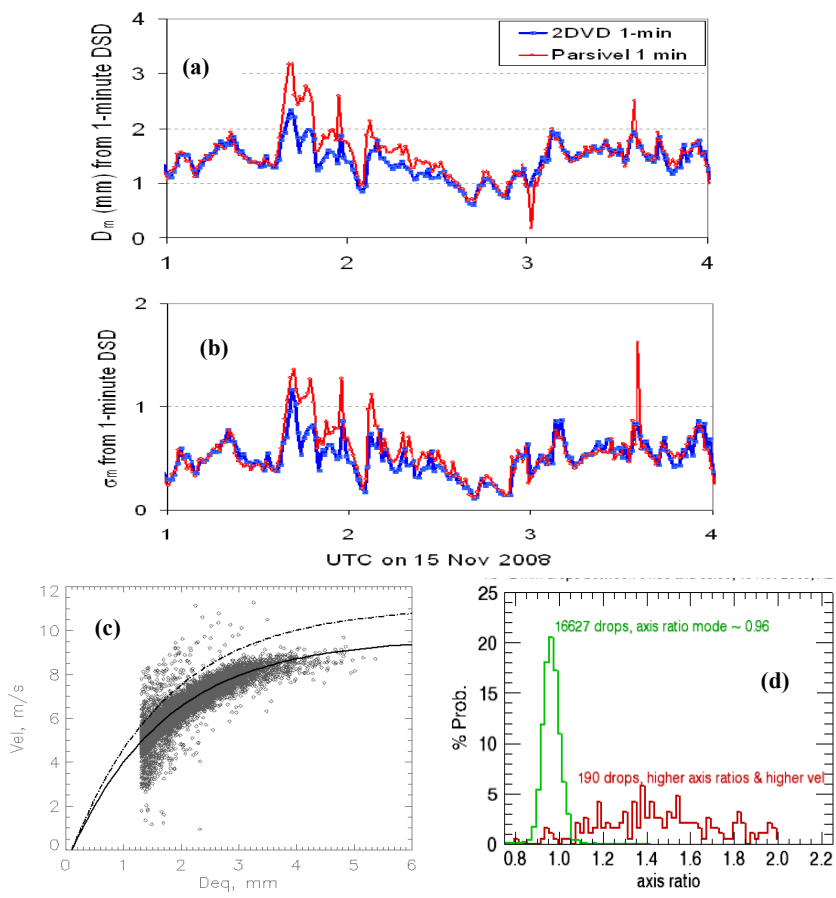

Fig. 2. (a and b): $D_{\mathrm{m}}$ and $\sigma_{\mathrm{m}}$ time series comparisons for the 15 November 2008 event. Descrepancies are seen between 01:40 UTC and 02:20 UTC. (c): Vertical velocity versus equivalent diameter for each hydrometeor measured for the 15 November 2008 event. The solid line shows the Atlas et al. (1973) variation and the dot-dashed line represents $15 \%$ above this variation. (d): Axis ratio distributions for all hydrometeors with $D$ in the $1.5-2.0 \mathrm{~mm}$ range in (c). The green histogram represents the distribution for all hydrometeors with fall speed below the $15 \%$ "elevated" curve (dot-dashed line) in (c) and the red histograms for all cases lying above the line.

History data for this event indicates moderate wind speed ( $\sim 8$ to $9 \mathrm{mph}$ ), but thunderstorm activity was reported and the Parsivel hydrometeor class indicator had "GR" flag, implying the possibility of small hail (non-fully-melted hydrometeors) or graupel, mixed in with fully-melted rain hydrometeors. The 2DVD based rain rates reached a maximum of $80 \mathrm{~mm} \mathrm{~h}^{-1}$ at around 01:45 UTC whereas Parsivel showed $120 \mathrm{~mm} \mathrm{~h}^{-1}$.

The fall velocity of individual hydrometeors from the 2DVD measurements for this event is shown in Fig. 2c as a scatter plot of the vertical velocity versus $D$ for all hydrometeors measured by the 2DVD for this event. Due to the limited instrument resolution, only the hydrometeors with $D>1.5 \mathrm{~mm}$ are shown. Superimposed on the scatter plot is the curve representing the velocity - diameter formula given by Atlas et al. (1973) curve as well as the same formula multiplied by $15 \%$. Most hydrometeors lie below the elevated curve but a small but significant number of them lie well above this curve, indicating the presence of small hail or graupel (or "ice pellets") with higher fall speeds. 
Since the 2DVD also measures the shape and the orientation of the individual hydrometeors, it was possible to examine the axis ratios of those lying above and below the $15 \%$ "elevated" curve. Figure 2d shows the axis ratio distributions for the drops with $D$ in the range $1.5-2 \mathrm{~mm}$, separated into the two categories. For the whole event, there were 16627 drops with fall speeds below the $15 \%$ elevated curve and 190 drops above it. For the former, the axis ratio distribution is narrow, with a clear mode at 0.96 , which is close to the previous measurements in rain (e.g. section 10.3 of Thurai and Bringi, 2008). On the other hand, the hydrometeors above the elevated curve give rise to a very broad axis ratio distribution with no clear peak, and in fact with axis ratios higher than one in many cases (indicating more prolate-like shapes). Clearly these are non-fully-melted hydrometeors.

As mentioned earlier, there are also considerable differences in the measurement techniques used by 2DVD and Parsivel. Aside from the fact that the 2DVD is a custombuilt research instrument while the Parsivel is more of an operational tool, there are other considerable differences in the measurement methods employed by the two instruments. The differences include: (a) via two parallel but verticallyseparated beams, the 2DVD provides a direct measure of drop fall speed whereas the Parsivel uses a dwell time within its single beam, (b) the 2DVD gives two orthogonal views of each drop from which the drop volume and equi-volume spherical diameter $\left(D_{\text {eq }}\right.$ or $D$ ) can be accurately computed (even in presence of horizontal component of the drop velocity) whereas the Parsivel gives the maximum dimension of the drop in only one plane, (c) the 2DVD computes N(D) using data from each drop whereas the Parsivel gives the concentration in a $32 \times 32$ matrix of velocity and diameter, and (d) the 2DVD computes rain rate as the volume flux over a sampling area twice that of the Parsivels. Out of these, only point (c) has been addressed previously in the literature, by Marzuki et al., 2010, who have shown, using simulations, that the "binning" of the data will not create any undue errors in $D_{\mathrm{m}}$. Regarding point (b) above, it is worth noting that - as found by Kinnell (1976) for the impact-type disdrometer - it is possible that Parsivel measurements may also be affected by shape and velocity variations for a given drop diameter.

\subsection{Statistical comparisons}

For statistical comparisons, we use here $D_{\mathrm{m}}, \sigma_{\mathrm{m}}$ and $R$ based on the 3-min moving average of the 1-min data, in order to reduce the "noisiness" arising from small-time scale temporal fluctuations (though a small amount of over-sampling may be introduced as a result). The scatter plot of $D_{\mathrm{m}}$ and $\sigma_{\mathrm{m}}$ from 2DVD and the Parsivel are shown in Fig. 3a, b respectively. A total of 4800 points are shown from all the events considered. While most of the points lie below $D_{\mathrm{m}}$ of $2 \mathrm{~mm}$ and the agreement there is good, note how beyond $2 \mathrm{~mm}$, there is a significant bias in the Parsivel values (overestimate at times by $20-30 \%$ ). This bias was evident at rainfall rates exceeding
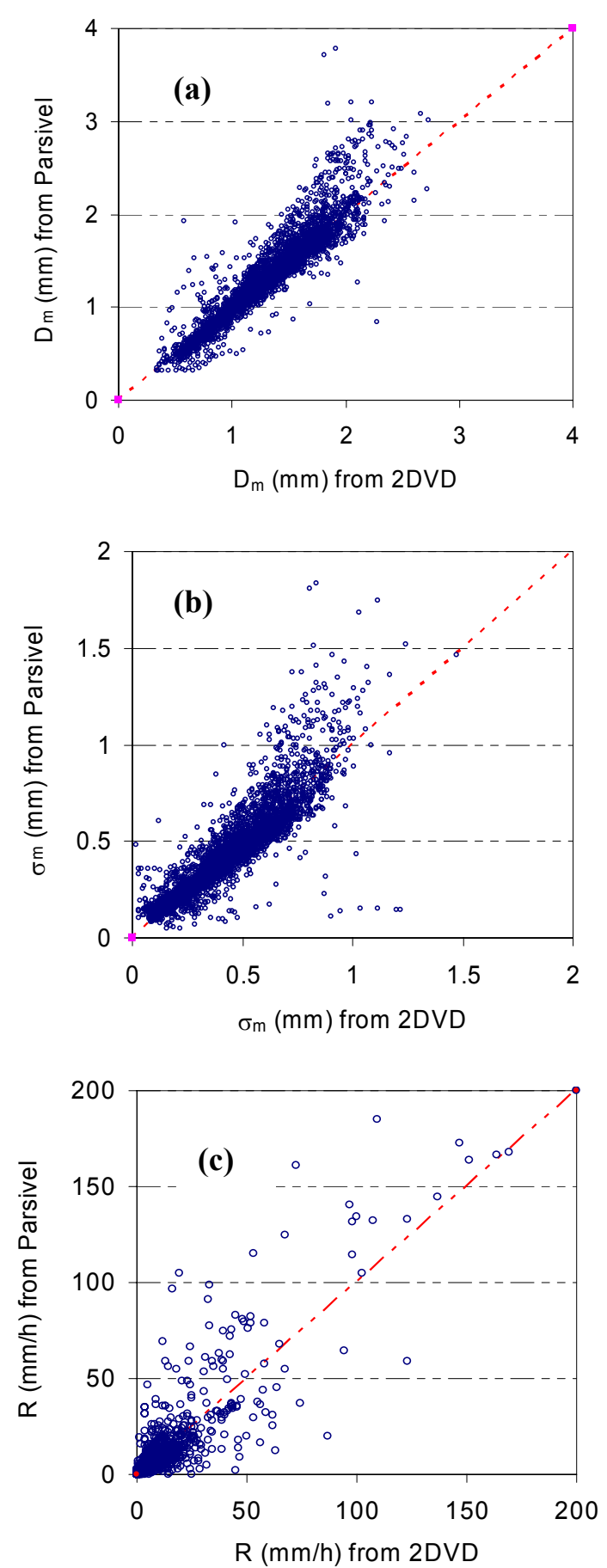

Fig. 3. Scatter plot of (a) $D_{\mathrm{m}}$ (b) $\sigma_{\mathrm{m}}$ and (c) $R$ between 2DVD and NSSTC Parsivel. Each data point represents a 3-min moving average of the 1-min data.

$20 \mathrm{~mm} \mathrm{hr}^{-1}$. The events used in the statistical comparisons are shown in Table 1, together with the duration and comparison of the rain accumulations with a co-located Geonor rain gauge, as well as the fractional bias and mean absolute deviation in $D_{\mathrm{m}}, \sigma_{\mathrm{m}}$ and $R$ between the 2DVD and Parsivel data. 
Table 1. The events used for statistical comparisons, together with rain accumulations, fractional bias, mean absolute standard deviation.

\begin{tabular}{|c|c|c|c|c|c|c|c|c|c|c|c|}
\hline Date & $\begin{array}{l}\text { Time } \\
\text { interval } \\
\text { (hours) }\end{array}$ & $\begin{array}{c}\text { Rain } \\
\text { accumulation } \\
\text { (2DVD) }\end{array}$ & $\begin{array}{c}\text { Rain } \\
\text { accumulation } \\
\text { (Parsivel) }\end{array}$ & $\begin{array}{c}\text { Rain } \\
\text { accumulation } \\
\text { (Geonor) }\end{array}$ & $\begin{array}{c}\text { Fractional } \\
\text { Bias (\%) } \\
\text { in } D_{\mathrm{m}}\end{array}$ & $\begin{array}{c}\text { Fractional } \\
\text { Bias (\%) } \\
\text { in } \sigma_{\mathrm{m}}\end{array}$ & $\begin{array}{c}\text { Fractional } \\
\text { Bias }(\%) \\
\quad \text { in } R\end{array}$ & $\begin{array}{c}\text { Mean absolute } \\
\text { deviation } \\
(\%) \text { of } D_{\mathrm{m}}\end{array}$ & $\begin{array}{c}\text { Mean absolute } \\
\text { deviation } \\
(\%) \text { of } \sigma_{\mathrm{m}}\end{array}$ & $\begin{array}{c}\text { Mean absolute } \\
\text { deviation } \\
(\%) \text { of } R\end{array}$ & $\begin{array}{c}\text { Includes } \\
\text { non-fully-melted } \\
\text { hydro-meteors }\end{array}$ \\
\hline 6 Jan 09 & $00-24$ & 73.9 & 64.5 & 76.6 & 3.1 & 7.6 & 13.7 & 4.8 & 10.6 & 34.5 & No \\
\hline 26 Mar 09 & $05-11$ & 41.8 & 43.1 & Not available & -7.2 & -9.5 & -3.1 & 9.7 & 15.9 & 48.8 & No \\
\hline 6 May 09 & $14-16$ & 23.8 & 23.0 & 27.6 & -0.4 & 0.9 & 5.7 & 6.8 & 11.1 & 23.7 & No \\
\hline 27 Feb 09 & $11-16$ & 22.4 & 17.6 & Not available & -1.6 & -0.3 & 10.8 & 5.7 & 12.5 & 25.1 & No \\
\hline 2 Apr 09 & $21-24$ & 48.3 & 54.3 & Not available & -8.6 & -7.2 & -12.5 & 12.5 & 15.7 & 33.6 & Yes \\
\hline 3 Apr 09 & 00-02 & 13.2 & 15.1 & Not available & -7.0 & -13.2 & -12.5 & 10.6 & 21.5 & 70.2 & No \\
\hline 14 Apr 09 & 02-08 & 8.2 & 6.7 & Not available & 3.5 & 12.2 & 24.2 & 4.0 & 13.4 & 44.0 & No \\
\hline 10 Apr 09 & $08-10$ & 11.6 & 10.5 & 14.3 & 0.9 & 3.3 & 10.3 & 7.1 & 13.5 & 76.6 & No \\
\hline 5 May 09 & 00-02 & 6.5 & 9.5 & 6.8 & -7.1 & 0.5 & -46.9 & 10.9 & 10.8 & 74.8 & No \\
\hline 15 Nov 08 & 01-04 & 24.0 & 27.6 & 24.7 & -9.6 & -9.6 & -14.8 & 11.1 & 16.3 & 51.3 & Yes \\
\hline 25 Aug 08 & $20-24$ & 11.1 & 9.5 & 8.0 & 1.8 & 7.2 & 13.7 & 3.3 & 9.3 & 15.2 & No \\
\hline 14 Jun 08 & 07-10 & 36.0 & 38.1 & 36.0 & -10.8 & -12.6 & -5.6 & 12.6 & 19.5 & 34.4 & Yes \\
\hline Average & & & & & -3.6 & -1.7 & -1.4 & 8.3 & 14.2 & 44.4 & \\
\hline
\end{tabular}

The table includes the 2 events considered earlier in Figs. 1 and 2. In general, the agreement between the 2DVD based rain accumulations and the Geonor measurements (whenever available) are seen to be closer than the corresponding values from the Parsivel data. Also shown in Table 1 (last row) is the average of the fractional bias and the mean absolute deviation. Negative biases are seen on average for all three parameters; also seen is the mean absolute deviation being the highest for $R$ and the lowest for $D_{\mathrm{m}}$.

Figure 3c shows scatter plots for $R$ estimated from the 2DVD and the Parsivel (here however, each data point is a 3-min block average of the base 1-min data). The agreement in $R$ is close for $R<\sim 20 \mathrm{~mm} \mathrm{~h}^{-1}$, but beyond that the Parsivel estimate tends to give higher values than the 2DVD. The correlation coefficient is 0.87 for the "side-by-side" placement of the 2 instruments. This value is considerably smaller than for "side-by-side" tipping bucket gages (0.95-0.97, e.g., Habib et al., 2001) which involved the 15-min averaged $R$. However, it is important to note that here, we are using different instruments with much smaller sampling areas and a 3-min averaged $R$ (to approximate the scale of a single radar pixel), so the lower correlation coefficient values are probably reasonable and more instrument-related as opposed to reflecting the natural variability of $R$ at very small spatial scales $(<$ several meters).

Relative to identifying a common measurement space for the two instruments as it pertains to DSD variability, we conclude from these comparisons (at a 3-min integration/smoothing scale) that the Parsivel instrument performs similar to the 2DVD for $D_{\mathrm{m}}<2 \mathrm{~mm}$ and/or for $R<$ $20 \mathrm{~mm} \mathrm{~h}^{-1}$ in terms of agreement with the 2DVD. The percentage of time that $D_{\mathrm{m}}>2 \mathrm{~mm}$ or $R>20 \mathrm{~mm} \mathrm{~h}^{-1}$ are, respectively, $6 \%$ and $4 \%$ (for our dataset comprising 4800 1-min data samples). The implication is that the Parsivel instruments can be used to evaluate DSD and rain rate variability at small spatial and temporal scales when the rain rate remains below approximately $20 \mathrm{~mm} \mathrm{~h}^{-1}$. However, as is well known, the contribution to rain accumulation from higher rain rates can be very significant depending on regime even though the frequency of occurrence can be very low. Other climatologies/regimes may yield different thresholds for $D_{\mathrm{m}}$ and/or $R$ where the Parsivel disdrometers would give reliable estimates.

\section{Parsivel-to-Parsivel and 2DVD-to-2DVD}

Values of $D_{\mathrm{m}}$ obtained from two "side-by-side" Parsivels were also compared (based on 2576 1-min samples). Their scatter plot is not shown here, but we report the following values: if we define $\Delta$ as the difference in the $\mathrm{D}_{m}$ values between the 2 instruments, the standard deviation of $\Delta$ (or, $\sigma_{\Delta}$ ) was $0.136 \mathrm{~mm}$ while the mean value of $D_{\mathrm{m}}$ (or, $\left.<D_{\mathrm{m}}\right\rangle$ ) from the 2 Parsivels is $1.47 \mathrm{~mm}$. Since the instruments were identical and the physical variations get cancelled out because of the "side-by-side" location, the sampling error of the Parsivel can be viewed in terms of the fractional standard deviation (FSD) $=\sigma_{\Delta} / \sqrt{ } 2 \div<D_{\mathrm{m}}>=6.57 \%$. Thus, the sampling error of the Parsivel for estimating $D_{\mathrm{m}}$ is considerably less than the expected physical variations in $D_{\mathrm{m}}$. "Side-by-side" comparisons of two 2DVD's (low profile and tall unit) by Cao et al. (2008) show similar sampling errors.

The scatter plot of $R$ between the two "side-by-side" Parsivels showed much higher correlation as compared with Parsivel versus 2DVD. The sampling error of the Parsivel disdrometer for estimating 3-min average $R$ in terms of the fractional standard error was found to be $36 \%$. This can be reduced by increasing the averaging time.

Side-by-side comparisons of two 2DVD's (low profile and the new compact unit) were also made. A total of 1723 1-min DSDs were used for the comparisons, and the correlation coefficients for $D_{\mathrm{m}}, \sigma_{\mathrm{m}}$ and $R$ were found to be $0.946,0.900$ and 0.978 respectively. The events include a cool-season tornadic storm which, despite the strong winds showed very close agreement between the two 2DVD instruments (see Thurai et al., 2010). 
Table 2. Correlation coefficients (and FSD of the sampling error).

\begin{tabular}{lccc}
\hline Parameter & Parsivel to 2DVD & 2DVD to 2DVD & Parsivel to Parsivel \\
\hline Rainrate & $0.903(75 \% *)$ & $0.98(14 \%)$ & $0.98(36 \%)$ \\
$D_{\mathrm{m}}$ & $0.851(10 \%)$ & $0.95(5 \%)$ & $0.96(6.6 \%)$ \\
$\sigma_{\mathrm{m}}$ & $0.834(15 \%)$ & $0.90(10.4 \%)$ & N/A \\
\hline
\end{tabular}

* reduces to $52 \%$ if $R$ is restricted to $1-12 \mathrm{~mm} \mathrm{~h}^{-1}$

Finally, in Table 2 we summarise the nugget parameters, namely, the correlation coefficients and the FSDs of the sampling error for the three-way inter-comparisons. Clearly, comparisons between the same type of instrument are better than their "cross comparisons". Although this is to be expected, it is possible that the Parsivel to 2DVD comparisons would improve if the comparisons were made exclusively for rain only cases, i.e. without any non-fully meted hydrometeors such as graupel, ice pellets or small hail.

\section{Summary and conclusions}

DSD comparisons between 2DVD and Parsivel have been made in terms of $D_{\mathrm{m}}, \sigma_{\mathrm{m}}$ and $R$ from the 1 min DSD measured by the two instruments. Time series comparisons showed close agreement in all three parameters for cases where $R$ was less than $20 \mathrm{~mm} \mathrm{~h}^{-1}$. In four cases, discrepancies in all three parameters were seen during part of the event, with Parsivel showing higher $D_{\mathrm{m}}, \sigma_{\mathrm{m}}$ and $R$, when $R$ reached high values (particularly over $30 \mathrm{~mm} \mathrm{~h}^{-1}$ ).

Scatter plots of $D_{\mathrm{m}}, \sigma_{\mathrm{m}}$ and $R$ based on the 1-min DSDs (time-corrected) were determined for all events. For the $D_{\mathrm{m}}$ comparisons, the majority of the points were below $2 \mathrm{~mm}$ and the agreement was very close. However for $D_{\mathrm{m}}>2 \mathrm{~mm}$, there was a significant bias in the Parsivel values (over estimate at times by $20-40 \%$ ). This bias was evident at rainfall rates exceeding $20 \mathrm{~mm} \mathrm{~h}^{-1}$.

Possible causes for the discrepancies in $D_{\mathrm{m}}, \sigma_{\mathrm{m}}$ and $R$ were investigated. In the four cases where significant discrepancies had been observed, the Parsivel measurements had indicated "GR" (or graupel) around the time of the deviation, and the "drop-by-drop" data from the 2DVD showed that whilst the majority of the hydrometeors followed the Gunn and Kinzer (1949) curve for the fall-velocity (to within $15 \%)$, there were some hydrometeors which had fall velocities significantly higher and with axis ratios very different from those expected for rain. Such non-fully-meltedhydrometeors, together with drop shape and velocity assumptions made by the Parsivel for large drops, are thought to contribute to the discrepancies seen.

For all cases considered, the correlation coefficients for $D_{\mathrm{m}}, \sigma_{\mathrm{m}}$ and $R$ were $0.90,0.85$ and 0.83 respectively, as shown in Table 2. The corresponding values for 2DVD-to2DVD comparisons were significantly higher, viz. 0.98, 0.95, and 0.90 respectively. For Parsivel-to-Parsivel the correlation coefficients were 0.98 for $R$ and 0.96 for $D_{\mathrm{m}}$. The fractional standard errors were also better for comparisons between the same type of instrument (e.g. for $D_{\mathrm{m}}, 5 \%$ for 2DVD and $6 \%$ for Parsivel) as compared with their cross-comparisons (10\% for 2DVD-to-Parsivel). These figures - representing the nugget parameters - need to be borne in mind when determining the spatial correlation from the dense disdrometer network datasets in the future.

Acknowledgements. MT acknowledges NASA Grant NNX09AD72G for funding this work. W. Petersen, P. Gatlin and C. Schultz gratefully acknowledge NASA PMM/GPM funding (via Ramesh Kakar, Arthur Hou, and Mathew Schwaller). The authors also wish to thank V. N. Bringi, L. Carey and W. Deierling for useful discussions.

Edited by: S. Michaelides and S. Athanasatos

Reviewed by: two anonymous referees

\section{References}

Atlas, D., Srivastava, R. C., and Sekkon, R. S.: Doppler radar characteristics of precipitation at vertical incidence, Rev. Geophys, Space GE, 2, 1-35, 1973.

Battaglia, A., Rustemeier, E., Tokay, A., Blahak, U., and Simmer, C.: Parsivel snow observations: a critical assessment, J. Atmos. Ocean. Tech., 27, 333-344, 2010.

Bringi, V. N., Williams, C. R., Thurai, M., and May, P. T.: Using Dual-Polarized Radar and Dual-Frequency Profiler for DSD Characterization: A Case Study from Darwin, Australia, J. Atmos. Ocean. Tech., 26, 2107-2122, 2009.

Cao, Q., Zhang, G., Brandes, E., Schuur, T., Ryzhkov, A., and Ikeda, K.: Analysis of video disdrometer and polarimetric radar data to characterize rain microphysics in Oklahoma, J. Appl. Meteorol., 47, 2238-2255, 2008.

Gunn, R. and G. Kinzer.: The terminal velocity of fall for water droplets in stagnant air, J. Meteorol., 6, 243-248, 1949.

Habib, E., Krajewski, W. F., and Ciach, G. J.: Estimation of interstation correlation coefficient in rainfall data, J. Hydrometeorol., 2, 621-629, 2001.

Hou, A. Y., Skofronick-Jackson, G., Kummerow, K., and Sheppard, J. M.: Global precipitation measurement, Chapter 6, "Precipitation: Advances in Measurement, Estimation and Prediction", edited by: Michaelides, S., Springer, ISBN: 978-3-540-77654-3, 2008.

Huang, G.-J., Bringi, V. N., and Thurai, M.: Orientation angle distributions of drops after an 80-m fall using a 2-D video disdrometer, J. Atmos. Ocean Tech., 25, 1717-1723, 2008.

Iguchi, T., Kozu, T., Kwiatkowski, J., Meneghini R., Awaka J., and Okamoto, K.: Uncertainties in the rain profiling algorithm for the TRMM precipitation radar, J. Meteorol. Soc. Japan, 87A, 1-30, 2009.

Kinnell, P. I. A.: Some observations on the Joss-Waldvogel rainfall disdrometer, J. Appl. Meteorol., 15, 499-502, 1976.

Kruger, A. and Krajewski, W.: Two-dimensional video disdrometer: a description. J. Atmos. Ocean. Tech. 19, 602-617, 2002. 
Löffler-Mang, M. and Joss, J.: An optical disdrometer for measuring size and velocity of hydrometeors, J. Atmos. Ocean. Tech. 17, 130-139, 2000.

Marzuki, M., Randeu, W. L., Schönhuber, M., Bringi, V. N., Kozu, T., and Shimomai, T.: Raindrop size distribution parameters of distrometer data with different bin sizes, IEEE Trans. Geosci. Remote, 48(8), 3075-3080, 2010.

Petersen, W. A., Knupp, K. R., Cecil, D. J., and Mecikalski, J. R.: The Unversity of Alabama Huntsville THOR Center instrumentation: Research and operational collaboration, Preprints, 33rd Int. Conf. on Radar Meteorology, AMS, Cairns, Australia, 2007.

Schönhuber, M., Lammer, G., and Randeu, W. L.: The 2D-videovistrometer, Chapter 1 in "Precipitation: Advances in Measurement, Estimation and Prediction", edited by: Michaelides, S., Springer, ISBN:978-3-540-77654-3, 2008.
Tapiador, F. J., Checa, R., and de Castro, M.: An experiment to measure the spatial variability of rain drop size distribution using sixteen laser disdrometers, Geoph. Res. Lett., 37, L16803, doi:10.1029/2010GL044120, 2010.

Thurai, M. and Bringi, V. N.: Rain microstructure from polarimetric radar and advanced disdrometers, Chapter 10 in "Precipitation: Advances in Measurement, Estimation and Prediction", edited by: Michaelides, S., Springer, ISBN:978-3-540-77654-3, 2008.

Thurai, M., Petersen, W. A., and Carey, L. D.: DSD characteristics of a cool-season tornadic storm using C-band polarimetric radar and two 2D-video disdrometers, in: Proceedings "The sixth European conference on Radar in meteorology and hydrology", Sibiu, Romania, September 2010, 2010.

Ulbrich, C. W. and Atlas, D.: Rainfall microphysics and radar properties: analysis methods for drop size spectra, J. Appl. Meteorol., 37, 912-923, 1998. 